\title{
Dipole Moment and High Resolution Rotational Spectrum of Fluorocyanoacetylene, FCCCN
}

\author{
Michael Krüger, Ilona Merke, and Helmut Dreizler \\ Abteilung Chemische Physik, Institut für Physikalische Chemie, Universität Kiel, Kiel, Germany \\ Z. Naturforsch. 47 a, $1241-1242$ (1992); received September 18, 1992

\begin{abstract}
The title molecule was produced in a dc discharge from pentafluoro benzonitrile, $\mathrm{C}_{6} \mathrm{~F}_{5} \mathrm{CN}$. The dipole moment of fluorocyanoacetylene was found to be $3.05(15) \mathrm{D}$ from the Stark effect. The ${ }^{14} \mathrm{~N}$ nuclear quadrupole coupling constant $\chi_{z z}=-4.2513(47) \mathrm{MHz}$ was determined from high resolution rotational spectra obtained with a pulsed nozzle molecular-beam microwave Fourier transform spectrometer.
\end{abstract}

Fluorocyanoacetylene, FCCCN, has become available several years ago [1]. Despite being relatively unstable at ambient temperature and the lack of a traditional synthetic route, several spectroscopic studies have been undertaken on this interesting molecule. The photoelectron [2] and mass [3] spectra have been obtained shortly after the synthesis of the title molecule, while the microwave spectrum in the ground and excited vibrational states [4] and the diode laser spectrum [5] of FCCCN have only recently been measured. In addition, a CNDO/2 study has been performed [6].

In this communication we report the electric dipole moment as determined from second order Stark effect and the ${ }^{14} \mathrm{~N}$ nuclear quadrupole coupling constant of FCCCN.

\section{Experimental}

Fluorocyanoacetylene has been produced in three different ways. A Tesla discharge (with a HF vacuumtester) in pentafluoro benzonitrile, $\mathrm{C}_{6} \mathrm{~F}_{5} \mathrm{CN}$, was used according to [1], the discharge products were trapped at $-196^{\circ} \mathrm{C}$ and then evaporated into a standard Stark modulated continuous-wave microwave (MW) spectrometer operated at low resolution. The yields were unsatisfactorily low and the half-lifetime of FCCCN in the brass absorption cell was below 0.5 minutes at room temperature.

In another experiment, $\mathrm{C}_{6} \mathrm{~F}_{5} \mathrm{CN}$ was subjected to a dc discharge (ca. $2.5 \mathrm{kV}, 3 \mathrm{~mA}$, platinum wire elec-

Reprint request to Prof. Dr. H. Dreizler, Abteilung Chemische Physik, Institut für Physikalische Chemie, Universität Kiel, Olshausenstr. 40, W-2300 Kiel 1, FRG. trodes at a distance of $10 \mathrm{~cm}$, pyrex discharge tube with $2.5 \mathrm{~cm}$ internal diameter, pressure approximately 100 mTorr) operated under flow conditions outside the MW absorption cell to generate a constant molecular signal with a signal to noise ratio between 10 and 100.

The high resolution MW spectrum was obtained with our molecular-beam microwave Fourier transform (MB-MWFT) spectrometer [7, 8]. In this spectrometer, FCCCN was produced in situ from $\mathrm{C}_{6} \mathrm{~F}_{5} \mathrm{CN}$ in a dc discharge inside the nozzle where a supersonic jet is generated upon expansion of a mixture of argon ( 0.5 bar $)$ and pentafluoro benzonitrile at its vapor pressure. The teflon pin of the valve was fitted with an electric connection in such a way that a wire protruded approximately $1 \mathrm{~mm}$ from the tip of the pin. A voltage of $+700 \mathrm{~V}$ was applied to the wire, using the stainless steel vacuum chamber of the spectrometer as the counter electrode. Upon opening of the valve and forming of the supersonic gas jet, the rising pressure in the expansion canal of the valve caused the ignition of the discharge between the wire and the nozzle plate (distance about $1 \mathrm{~mm}$ ). The sharp pressure drop after closing of the valve ended the discharge. Due to inefficient production of FCCCN (at least in its vibrational ground state!) in this pulsed valve - pulsed discharge assembly, several thousand experiment cycles were averaged (measuring time ca. 10-20 minutes).

\section{Results}

The dipole moment was determined from the data given in Table 1 to be 3.05 (15) $\mathrm{D}$ from the second

0932-0784 / 92 / 1200-1241 \$01.30/0. - Please order a reprint rather than making your own copy. 


\begin{tabular}{ll}
\hline$M_{J}$ & $\Delta v$ \\
\hline 1 & $-3.65(20)$ \\
2 & $-1.41(20)$ \\
3 & $+2.44(20)$ \\
4 & $+8.12(20)$ \\
\hline
\end{tabular}

Table 1. Stark effect of the rotational transition $J+1 \leftarrow J$ with $J=5$ of fluorocyanoacetylene, FCCCN. Applied electric field strength $1800 \mathrm{~V} \mathrm{~cm}^{-1}$. $M_{J}$ : Projection quantum number of the Stark satellite; $\Delta v$ : frequency displacement from zero field transition frequency $(\mathrm{MHz})$.

Table 2. Hyperfine structure in the rotational spectrum of fluorocyanoacetylene, FCCCN. $J, F$ : Rotational and hyperfine quantum numbers of the transitions $J+1 \leftarrow J, F+1 \leftarrow F$; $v$ : observed transition frequency $(\mathrm{MHz})$. The difference $\Delta v$ $(\mathrm{kHz})$ between observed and calculated frequencies are below $0.35 \mathrm{kHz}$ in all cases. The standard error $\sigma$ of the fit is $\sigma=0.28 \mathrm{kHz}$

\begin{tabular}{llrr}
\hline$J$ & $F$ & $v$ & \multicolumn{1}{c}{$\Delta v$} \\
\hline 1 & 1 & 8283.8273 & -0.17 \\
1 & 2 & 8283.9188 & 0.20 \\
2 & 1 & 12425.5232 & 0.15 \\
2 & 2 & 12425.7358 & 0.13 \\
2 & 3 & 12425.7860 & -0.30 \\
3 & 2 & 16567.5459 & -0.20 \\
3 & 3 & 16567.6371 & -0.13 \\
3 & 4 & 16567.6698 & 0.35 \\
\hline
\end{tabular}

order Stark effect. The spectrometer was calibrated with the dipole moment of carbonyl sulfide (OCS) $\mu=0.715196$ (10) D [9].

The nuclear quadrupole coupling constant of ${ }^{14} \mathrm{~N}$ was extracted from the high resolution data given in Table 2 and found to be $\chi_{z z}=-4.2513$ (47) MHz. The ${ }^{14} \mathrm{~N}$ spin-rotation constant could not be determined from the spectra. Effects of ${ }^{19} \mathrm{~F}$ spin-rotation interaction were not noticed, but were possibly covered by noise. The ground state rotational constant $B_{0}=$
2070.957606 (47) MHz and centrifugal distortion constant $D=92.2$ (18) were fitted simultaneously with our program SIM2QS [10]. They agree reasonably with $B_{0}=2070.95708$ (59) $\mathrm{MHz}$ and $D=90.32$ (95) Hz from [4]. Because the number of measured lines is not very large the error distribution will not necessarily be normal. Due to the fact that a least squares fit implies a Gaussian distribution, the obtained standard errors are only formal and not reliable in the sence of a $67 \%$ confidence limit. Unfortunately the measurement of additional lines was not feasible for us.

Attempts to measure rotational transitions of isotopic species, thus allowing the determination of structural parameters, failed with both the Stark and MB-MWFT spectrometers due to insufficient signal intensities. Furthermore, the determination of a complete set of bond lengths of FCCCN from ground state rotational constants will be difficult because there are no other stable isotopes of fluorine and the central carbon atom is located extremely close to the center of gravity of the molecule.

After having completed this work we noticed that Ogata et al., Vancouver, investigated independently the rotational spectrum of this molecule.

\section{Acknowledgements}

We would like to thank Dagmar Preugschat and Dr. D. Lentz, Berlin, for advice, Dr. W. Stahl and J.-U. Grabow for their assistance with the molecular beam spectrometer and for interesting discussions. We gratefully acknowledge funding from the Deutsche Forschungsgemeinschaft, the Fonds der Chemie and the Land Schleswig-Holstein.
[1] G. Bieri, J. P. Stadelmann, F. Thommen, and J. Vogt, Helv. Chim. Acta 61, 357 (1978).

[2] G. Bieri, E. Heilbronner, V. Hornung, E. Kloster-Jensen, J. P. Maier, and W. von Niessen, Chem. Phys. 36, 1 (1979).

[3] U. Buechler and J. Vogt, Org. Mass. Spectrom. 14, 503 (1979).

[4] K. Tanaka, T. Okabayashi, and T. Tanaka, J. Mol. Spectrosc. 132, 467 (1988).

[5] M. Niedenhoff, K. M. T. Yamada, and G. Winnewisser, J. Mol. Spectrosc. 145, 290 (1991).
[6] R. W. Taft and R. T. C. Brownlee, J. Amer. Chem. Soc. 92, 7007 (1970).

[7] U. Andresen, H. Dreizler, J.-U. Grabow, and W. Stahl, Rev. Sci. Instrum. 61, 3694 (1990).

[8] J.-U. Grabow and W. Stahl, Z. Naturforsch. 45a, 1043 (1990).

[9] K. Tanaka, H. Ito, K. Harada, and T. Tanaka, J. Chem. Phys. 80, 5893 (1984).

[10] Program SYM2QS, author J. Gripp, Kiel. 